\title{
Identification of the Distribution and Sources of Pu in the Northern South China
}

\section{Sea: Influences of Provenance and Scavenging}

RUIRUI WANG ${ }^{\dagger}$, LING LEI $^{\dagger}$, GANG LI* ${ }^{*}$, ZHIYONG LIU* ${ }^{*} \dagger$

$\uparrow$ State Key Laboratory of Radiation Medicine and Protection, School for Radiological and Interdisciplinary Sciences (RAD-X) and Collaborative Innovation Centre of Radiation Medicine of Jiangsu Higher Education Institutions, Soochow University, Suzhou 215123, China.

\$ Key Laboratory of Ocean and Marginal Sea Geology, South China Sea Institute of Oceanology, Chinese Academy of Sciences, Guangzhou 510300, China.

* Corresponding authors. Email: gangli@scsio.ac.cn (GANG LI); liuzy@suda.edu.cn (ZHIYONG LIU); Tel: 0086-512-65883945; Fax: 0086-512-65880035. 


\section{Table S1}

Analytical results of ${ }^{239+240} \mathrm{Pu}$ activities and ${ }^{240} \mathrm{Pu} /{ }^{239} \mathrm{Pu}$ atom ratios.

$\begin{array}{lccccc}\text { Sample } & \text { Longitude } & \text { Latitude } & \text { depth }(\mathrm{m}) & { }^{239+240} \mathrm{Pu} & { }^{240} \mathrm{Pu} /{ }^{239} \mathrm{Pu} \\ \text { SC01 } & 111.26 & 19.83 & 58.3 & 0.657 \pm 0.110 & 0.257 \pm 0.015 \\ \text { SC02 } & 111.26 & 20.20 & 56.6 & 0.963 \pm 0.109 & 0.293 \pm 0.017 \\ \text { SC03 } & 111.26 & 20.38 & 53.4 & 1.428 \pm 0.093 & 0.275 \pm 0.016 \\ \text { SC04 } & 111.08 & 21.09 & 26.2 & 0.443 \pm 0.050 & 0.276 \pm 0.016 \\ \text { SC05 } & 111.93 & 21.55 & 22.1 & 0.389 \pm 0.039 & 0.285 \pm 0.017 \\ \text { SC06 } & 111.31 & 20.38 & 57.3 & 1.718 \pm 0.111 & 0.271 \pm 0.016 \\ \text { SC07 } & 111.22 & 20.38 & 49.7 & 0.639 \pm 0.029 & 0.273 \pm 0.016 \\ \text { SC08 } & 111.40 & 20.83 & 43.9 & 0.566 \pm 0.017 & 0.271 \pm 0.016 \\ \text { SC09 } & 112.08 & 21.19 & 40.7 & 0.824 \pm 0.073 & 0.272 \pm 0.016 \\ \text { SC10 } & 111.99 & 20.88 & 52.7 & 0.479 \pm 0.021 & 0.272 \pm 0.016 \\ \text { SC11 } & 111.94 & 20.97 & 49.1 & 1.078 \pm 0.157 & 0.277 \pm 0.016 \\ \text { SC12 } & 111.85 & 21.15 & 39.4 & 0.421 \pm 0.033 & 0.279 \pm 0.016 \\ \text { SC13 } & 113.39 & 21.94 & 14.5 & 0.356 \pm 0.027 & 0.264 \pm 0.015 \\ \text { SC14 } & 113.42 & 21.99 & 3.6 & 0.178 \pm 0.030 & 0.250 \pm 0.015\end{array}$




\begin{tabular}{|c|c|c|c|c|c|}
\hline $\mathrm{SC} 15$ & 113.20 & 21.94 & 9.6 & $0.079 \pm 0.011$ & $0.216 \pm 0.013$ \\
\hline SC16 & 112.96 & 21.86 & 28.4 & $0.040 \pm 0.019$ & $0.263 \pm 0.015$ \\
\hline SC17 & 114.31 & 21.53 & 71.7 & $1.313 \pm 0.156$ & $0.285 \pm 0.017$ \\
\hline $\mathrm{SC} 18$ & 114.67 & 21.53 & 73.4 & $1.234 \pm 0.105$ & $0.281 \pm 0.015$ \\
\hline SC19 & 114.03 & 21.35 & 68.7 & $1.149 \pm 0.107$ & $0.292 \pm 0.017$ \\
\hline $\mathrm{SC} 20$ & 112.26 & 21.38 & 29.5 & $1.052 \pm 0.175$ & $0.296 \pm 0.017$ \\
\hline $\mathrm{SC} 21$ & 110.93 & 20.84 & 29.4 & $0.649 \pm 0.053$ & $0.257 \pm 0.015$ \\
\hline $\mathrm{SC} 22$ & 110.81 & 21.13 & 17.3 & $0.428 \pm 0.034$ & $0.231 \pm 0.013$ \\
\hline $\mathrm{SC} 23$ & 111.14 & 20.97 & 29.9 & $0.670 \pm 0.070$ & $0.266 \pm 0.015$ \\
\hline $\mathrm{SC} 24$ & 111.30 & 21.20 & 26.5 & $0.296 \pm 0.015$ & $0.244 \pm 0.014$ \\
\hline $\mathrm{SC} 25$ & 111.51 & 21.32 & 26.1 & $0.624 \pm 0.044$ & $0.264 \pm 0.015$ \\
\hline $\mathrm{SC} 26$ & 111.56 & 21.20 & 32.6 & $0.934 \pm 0.052$ & $0.259 \pm 0.015$ \\
\hline $\mathrm{SC} 27$ & 112.17 & 21.58 & 8.6 & $0.166 \pm 0.009$ & $0.232 \pm 0.014$ \\
\hline $\mathrm{SC} 28$ & 112.58 & 20.49 & 82.5 & $0.498 \pm 0.260$ & $0.277 \pm 0.016$ \\
\hline $\mathrm{SC} 29$ & 112.15 & 21.04 & 47.2 & $0.918 \pm 0.058$ & $0.280 \pm 0.016$ \\
\hline $\mathrm{SC} 30$ & 114.60 & 19.40 & 1109.5 & $0.865 \pm 0.032$ & $0.820 \pm 0.012$ \\
\hline SC31 & 111.02 & 19.33 & 65.9 & $1.113 \pm 0.125$ & $0.277 \pm 0.016$ \\
\hline $\mathrm{SC} 32$ & 111.23 & 18.40 & 194.7 & $1.847 \pm 0.327$ & $0.256 \pm 0.015$ \\
\hline SC33 & 110.28 & 18.38 & 78.1 & $0.614 \pm 0.214$ & $0.305 \pm 0.018$ \\
\hline $\mathrm{SC} 34$ & 114.38 & 21.84 & 50.1 & $1.496 \pm 0.057$ & $0.272 \pm 0.016$ \\
\hline SC35 & 113.73 & 22.30 & 8.2 & $0.073 \pm 0.012$ & $0.229 \pm 0.013$ \\
\hline SC36 & 113.70 & 22.25 & 7.5 & $0.242 \pm 0.02$ & $0.227 \pm 0.013$ \\
\hline $\mathrm{SC} 37$ & 113.76 & 22.17 & 8.7 & $0.286 \pm 0.08$ & $0.262 \pm 0.015$ \\
\hline SC38 & 113.79 & 22.38 & 11.2 & $0.202 \pm 0.021$ & $0.205 \pm 0.012$ \\
\hline
\end{tabular}




$\begin{array}{lrrccc}\text { SC39 } & 111.05 & 18.72 & 127.9 & 1.539 \pm 0.367 & 0.275 \pm 0.016 \\ \text { SC40 } & 110.94 & 18.99 & 98.6 & 1.589 \pm 0.083 & 0.259 \pm 0.015 \\ \text { SC41 } & 115.50 & 19.51 & 2397.6 & 0.506 \pm 0.026 & 0.245 \pm 0.014 \\ \text { SC42 } & 110.50 & 17.99 & 141.6 & 1.822 \pm 0.206 & 0.276 \pm 0.016 \\ \text { SC43 } & 114.00 & 20.99 & 82.3 & 0.262 \pm 0.014 & 0.276 \pm 0.016 \\ \text { SC44 } & 114.89 & 19.50 & 1548.4 & 0.357 \pm 0.047 & 0.281 \pm 0.015\end{array}$

\section{Table S2}

Sediment components of surface sediments.

\begin{tabular}{|c|c|c|c|}
\hline Site & Clay $(\%)^{\mathrm{a}}$ & Silt $(\%)^{b}$ & Sand $(\%)^{\mathrm{c}}$ \\
\hline SC01 & 6 & 27 & 67 \\
\hline $\mathrm{SC} 02$ & 12 & 63 & 25 \\
\hline $\mathrm{SC} 03$ & 17 & 66 & 17 \\
\hline $\mathrm{SC} 04$ & 13 & 45 & 42 \\
\hline $\mathrm{SC} 05$ & 20 & 75 & 5 \\
\hline SC06 & 17 & 66 & 17 \\
\hline $\mathrm{SC} 07$ & 17 & 66 & 17 \\
\hline SC08 & 16 & 70 & 14 \\
\hline SC09 & 11 & 56 & 33 \\
\hline SC10 & 10 & 50 & 40 \\
\hline SC11 & 12 & 56 & 32 \\
\hline $\mathrm{SC} 12$ & 11 & 51 & 38 \\
\hline $\mathrm{SC} 13$ & 12 & 86 & 2 \\
\hline $\mathrm{SC} 14$ & 8 & 58 & 33 \\
\hline
\end{tabular}




\begin{tabular}{|c|c|c|c|}
\hline $\mathrm{SC} 15$ & 11 & 86 & 3 \\
\hline $\mathrm{SC} 16$ & 10 & 56 & 34 \\
\hline $\mathrm{SC} 17$ & 6 & 75 & 19 \\
\hline SC18 & 7 & 69 & 24 \\
\hline SC19 & 6 & 61 & 33 \\
\hline SC20 & 13 & 59 & 28 \\
\hline $\mathrm{SC} 21$ & 8 & 50 & 43 \\
\hline $\mathrm{SC} 22$ & 10 & 48 & 42 \\
\hline $\mathrm{SC} 23$ & 10 & 65 & 25 \\
\hline $\mathrm{SC} 24$ & 15 & 56 & 29 \\
\hline $\mathrm{SC} 25$ & 8 & 64 & 27 \\
\hline $\mathrm{SC} 26$ & 17 & 59 & 24 \\
\hline $\mathrm{SC} 27$ & 11 & 66 & 23 \\
\hline $\mathrm{SC} 28$ & 2 & 17 & 82 \\
\hline $\mathrm{SC} 29$ & 11 & 52 & 37 \\
\hline SC30 & 14 & 66 & 19 \\
\hline SC31 & 9 & 34 & 56 \\
\hline SC32 & 14 & 66 & 19 \\
\hline SC33 & 8 & 31 & 61 \\
\hline SC34 & 14 & 61 & 25 \\
\hline SC35 & 17 & 77 & 6 \\
\hline SC36 & 15 & 78 & 6 \\
\hline SC37 & 12 & 69 & 18 \\
\hline SC38 & 15 & 76 & 8 \\
\hline
\end{tabular}




$\begin{array}{lccc}\text { SC39 } & 13 & 64 & 24 \\ \mathrm{SC} 40 & 12 & 44 & 44 \\ \mathrm{SC} 41 & 15 & 68 & 17 \\ \mathrm{SC} 42 & 73 & 13 \\ \mathrm{SC} 43 & 14 & 15 & 83 \\ \mathrm{SC} 44 & 2 & 69 & 15\end{array}$

Diameter ranges: a, clay particles, $0.02 \mu \mathrm{m}$ to $64 \mu \mathrm{m}$; b, silt particles, $64 \mu \mathrm{m}$ to $128 \mu \mathrm{m}$; c, sand particles, $128 \mu \mathrm{m}$ to $2000 \mu \mathrm{m}$. 


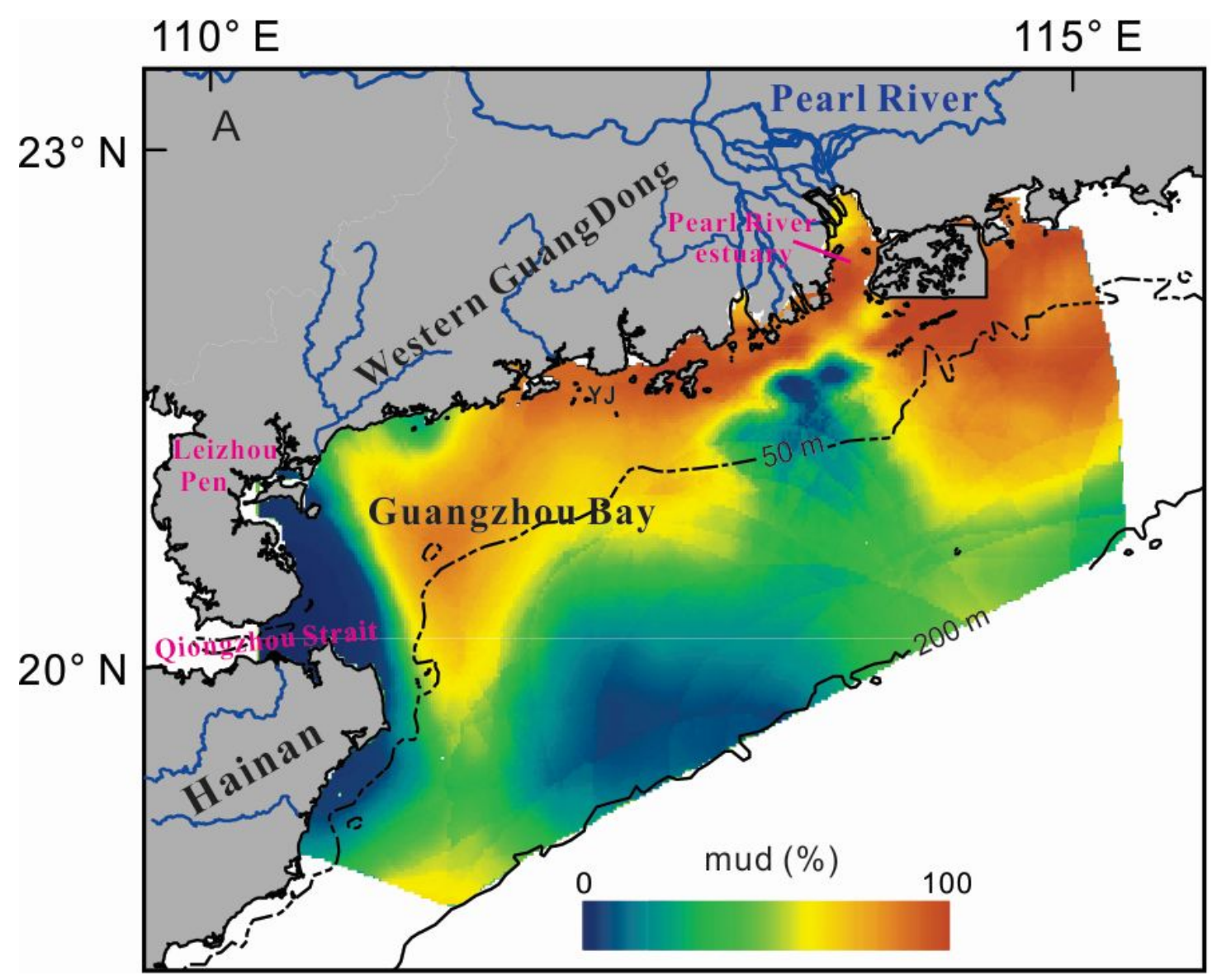

Fig. S1. The study area and the sediment compositions (unpublished results). 


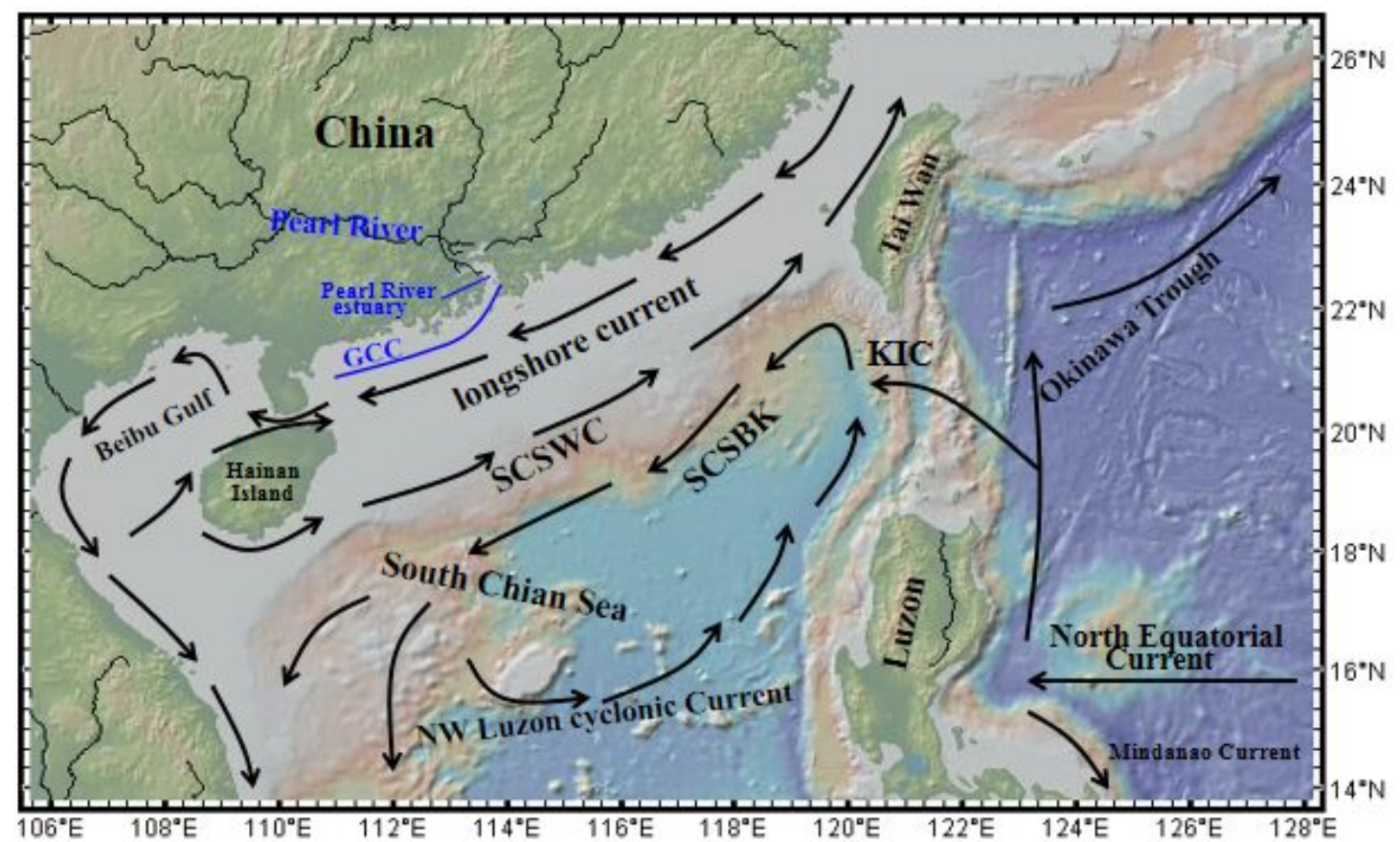

Fig. S2. Map of Chinese marginal seas and the shelf circulation patterns ${ }^{1-8}$.

Abbreviations in the figure are as follows: KIC, Kuroshio intrusion current; SCSBK, SCS branch of the Kuroshio Current; longshore current; NW Luzon Coastal Current; SCS Warm Current; GCC, Guangdong Coastal Current.

\section{References}

(1) $\mathrm{Su}$, C. C.; Huh, C. A. ${ }^{210} \mathrm{~Pb},{ }^{137} \mathrm{Cs}$ and ${ }^{239+240} \mathrm{Pu}$ in East China Sea sediments: sources, pathways and budgets of sediments and radionuclides. Mar. Geol. 2002, 183, $163-178$

(2) Liu, Y.; Gao, S.; Wang, Y. P.; Yang, Y.; Long, J.; Zhang, Y.; Wu, X. Distal mud deposits associated with the Pearl River over the northwestern continental shelf of the South China Sea. Mar. Geol. 2014, 347, 43-57.

(3) Bertine, K. K.; Chow, T. J.; Koide, M.; Goldberg, E. D. Plutonium isotopes in the environment: some existing problems and some new ocean results. J. Environ. Radioactiv. 1986, 3(3): 189-201. 
(4) Norisuye, K.; Okamura, K.; Sohrin, Y.; Hasegawa, H.; Nakanishi, T. Large volume preconcentration and purification for determining the ${ }^{240} \mathrm{Pu} /{ }^{239} \mathrm{Pu}$ isotopic ratio and ${ }^{238} \mathrm{Pu} /{ }^{239+240} \mathrm{Pu}$ alpha-activity ratio in seawater. J. Radioanal. Nucl. Ch. 2005, 267(1): 183-193.

(5) Yamada, M.; Zheng, J.; Wang, Z. L. ${ }^{137} \mathrm{Cs},{ }^{239+240} \mathrm{Pu}$ and ${ }^{240} \mathrm{Pu} / 239 \mathrm{Pu}$ atom ratios in the surface waters of the western North Pacific Ocean, eastern Indian Ocean and their adjacent seas. Sci. Total. Environ. 2006, 366(1): 242-252.

(6) Yamada, M.; Zheng, J.; Wang, Z. L. ${ }^{240} \mathrm{Pu} /{ }^{239} \mathrm{Pu}$ atom ratios in seawater from Sagami Bay, western Northwest Pacific Ocean: sources and scavenging. J. Environ. Radioactiv. 2007, 98(3): 274-284.

(7) Yamada, M.; Zheng, J. Determination of ${ }^{240} \mathrm{Pu} /{ }^{239} \mathrm{Pu}$ atom ratio in coastal surface seawaters from the western North Pacific Ocean and Japan Sea. Appl. Radiat. Isotopes. 2008, 66(1): 103-107.

(8) Yamada, M.; Zheng, J. Determination of ${ }^{240} \mathrm{Pu} /{ }^{239} \mathrm{Pu}$ atom ratio in seawaters from the East China Sea. Radiat. Prot. Dosim. 2011, 146(1-3): 311-313.

(9) Yamada, M.; Zheng, J. Temporal variation of ${ }^{240} \mathrm{Pu} /{ }^{239} \mathrm{Pu}$ atom ratio and ${ }^{239+240} \mathrm{Pu}$ inventory in water columns of the Japan Sea. Sci. Total. Environ. 2010, 408(23): 5951-5957. 